ESAIM: M2AN

Vol. 41, No 2, 2007, pp. 333-350

DOI: $10.1051 / \mathrm{m} 2 \mathrm{an}: 2007019$
ESAIM: Mathematical Modelling and Numerical Analysis

www.edpsciences.org/m2an

\title{
MOLECULAR SIMULATION IN THE CANONICAL ENSEMBLE AND BEYOND
}

\author{
ZHIDONG JiA ${ }^{1}$ AND BEN LEIMKUHLER ${ }^{2}$
}

\begin{abstract}
In this paper, we discuss advanced thermostatting techniques for sampling molecular systems in the canonical ensemble. We first survey work on dynamical thermostatting methods, including the Nosé-Poincaré method, and generalized bath methods which introduce a more complicated extended model to obtain better ergodicity. We describe a general controlled temperature model, projective thermostatting molecular dynamics (PTMD) and demonstrate that it flexibly accommodates existing alternative thermostatting methods, such as Nosé-Poincaré, Nosé-Hoover (with or without chains), Bulgac-Kusnezov, or recursive Nosé-Poincaré Chains. These schemes offer possible advantages for use in computing thermodynamic quantities, and facilitate the development of multiple time-scale modelling and simulation techniques. In addition, PTMD advances a preliminary step toward the realization of true nonequilibrium motion for selected degrees of freedom, by shielding the variables of interest from the artificial effect of thermostats. We discuss extension of the PTMD method for constant temperature and pressure models. Finally, we demonstrate schemes for simulating systems with an artificial temperature gradient, by enabling the use of two temperature baths within the PTMD framework.
\end{abstract}

Mathematics Subject Classification. 74A25, 82C80.

Received: October 31, 2005.

\section{INTRODUCTION}

A flexible computational modelling approach is needed for simulating a wide range of problems in biology, chemistry and materials science. For the most challenging applications, development of numerical methods must go hand in hand with improved formulation of the models themselves, as the properties of the equations may have profound effect on the properties of available numerical methods for their treatment. This type of comprehensive model development is not often undertaken. For example, there is at this point in time no widespread agreement on the correct way to formulate the numerical-dynamical procedure for coarse-grained molecular models, although this is of increasing importance in studies of crack propagation and nanoindentation [26,35]. The computation of free energy profiles for complex macromolecules, e.g. membrane proteins, is likewise still in the ground state, despite a variety of intriguing proposals (see, e.g., [10] for a recent survey). If we are going to push scale regimes forward to treat macromolecular systems on timescales longer than a few nanoseconds, a new approach will need to be developed.

\footnotetext{
Keywords and phrases. Molecular dynamics, constant temperature, thermostat, barostat, nonequilibrium simulation.

1 ICMSEC, P.O. Box 2719, Beijing 100080, China.

2 School of Mathematics and Maxwell Institute, University of Edinburgh, Edinburgh EH9 3JZ, UK. b.leim@ntlworld.com
} 
In this article, we focus on one particular challenge - developing a framework for partially thermostatted molecular dynamics. We first survey our recent work aimed at designing better projective dynamical thermostatting methods based on extended Hamiltonians and associated volume preserving, time-reversible integrators. We then propose an alternative formulation for pre-equilibrium modelling of systems with a temperature gradient as discussed in [17]. Some preliminary experiments are also included. The emphasis in our proposed formulation is to prepare the ground for multiscale numerics.

The rest of this article is organized as follows. In Section 2, we discuss several variants of Nosé dynamics which replace the Newtonian dynamical formalism with an extended phase space model whose trajectories in the original phase space variables sample the canonical ensemble. Many different extensions are possible and we survey several. One of the limitations of Nosé dynamics is the need to assume the ergodicity which may or may not be present in the dynamics; in Section 3, we show how extended Hamiltonian models can be designed to improve the extent to which the underlying phase space is covered by trajectories, i.e. models with "enhanced ergodicity". In Section 4, we discuss projective thermostatting which allows for a more flexible approach to complex models, also extending the formalism for constant temperature and pressure simulation. Finally Section 5 discusses the prospects for several independent thermostats to be used for different variables, or even the possibility of nonequilibrium simulation of a system with an artificially enforced thermal gradient; a preliminary numerical experiment is included.

\section{NosÉ-STYle DYNAMiCS}

The constant temperature (also termed canonical, or Gibbs) ensemble can be sampled using Monte Carlo methods [13], and stochastic Langevin-type dynamics (including dissipative particle dynamics) [12,33], as well as deterministic extended dynamics techniques, which are the focus of this paper. Nosé's discovery [29], that one can sample the canonical ensemble by extending the dynamics with as little as one additional thermal degree of freedom (under certain circumstances), has found widespread applicability in a variety of systems ranging from atomistic models of materials to biomolecules.

The key idea suggested by Nosé (based, in part, on related ideas of Andersen [1] for handling constant pressure) is that we can augment the energy function by incorporating a single additional phase variable, $s$, together with its canonical momentum, $\pi$, in the following way:

$$
H^{\text {Nosé }}=H(q, \tilde{p} / s)+\frac{p_{s}^{2}}{2 \mu}+g k T \ln s .
$$

A simple integration argument can be used to show that

$$
\int \delta\left(H^{\text {Nosé }}(q, \tilde{p}, s, \pi)-E\right) \mathrm{d} s \mathrm{~d} \pi \mathrm{d} q \mathrm{~d} \tilde{p}=\text { constant } \times \int \exp \left(-\frac{N+1}{g k T} H(q, p)\right) \mathrm{d} q \mathrm{~d} p,
$$

moreover,

$$
\begin{aligned}
& \int f(q, \tilde{p} / s) \delta\left(H^{\text {Nosé }}(q, \tilde{p}, s, \pi)-E\right) \mathrm{d} s \mathrm{~d} \pi \mathrm{d} q \mathrm{~d} \tilde{p} \\
& =\text { constant } \times \int f(q, p) \exp \left(-\frac{N}{g k T} H(q, p)\right) \mathrm{d} q \mathrm{~d} p
\end{aligned}
$$

where $p=\tilde{p} / s$. So if we choose $g=N+1$, we are able to reduce the microcanonical density function for $H^{\text {Nosé }}$ to the canonical one for $H$. Nosé's device has raised many additional questions and issues related to time scale, the modelling of rare events and the ergodic behavior.

This idea, as described, is in fact too simple. In practice this approach does not work reliably for simulation without further adjustment. But the basic integration argument is nonetheless quite remarkable. The reason that it works is the same reason that we are able to write the canonical probability density function in its very 

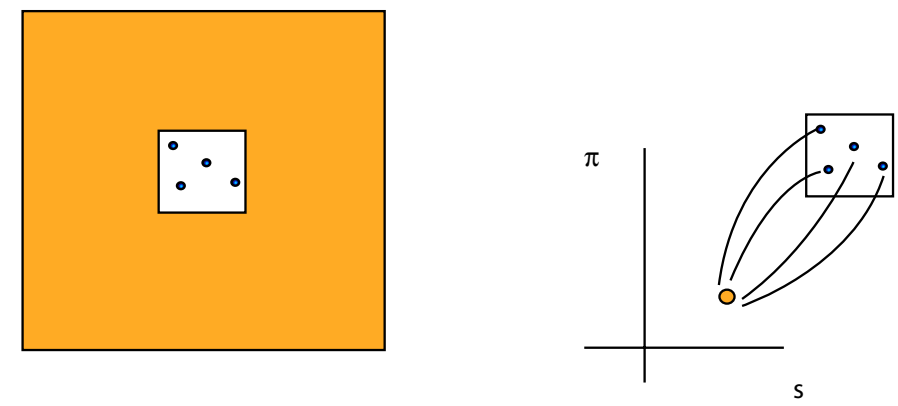

Figure 1. A heat bath is diagrammed at left, a large collection of physical particles which interact with the system. In Nosé dynamics this complicated bath is replaced by a single artificial variable, a "global demon" which simultaneously contacts all the physical degrees of freedom.

simple form: many microcanonical extensions of a given system would naturally reduce to the same canonical density function after integration over the extraneous variables. In Nosé's model, we add a temperature bath, but this bath has only one degree of freedom and yet the procedure often has the same practical result as one would obtain by introducing a much more complicated and more realistic atomistic bath. The use of a single degree of freedom which interacts with all real variables is a fundamentally nonphysical concept: the Nosé variable interacts in a nonlocal way with the system variables. For this reason, it has been referred to as a "global demon" by Kusnezov [22].

\subsection{Time Rescaling in Nosé dynamics: the Nosé-Poincaré method}

The so-called real variable Nosé-Hoover (NH) dynamics [16] is based on simple coordinate and time transformations of the dynamics subject to (2.1); this formulation reveals the underlying differential feedback mechanism of thermal control and makes it easy to recover data at equally spaced points in time. The fundamental benefit of the time-rescaling is however related to stability of computational methods.

In the dynamics associated to the Hamiltonian (2.1), the coordinate equation is written as

$$
\dot{q}=\frac{M^{-1} p}{s^{2}} .
$$

If $p / s$ is properly bounded due to the equipartition theorem, then the additional factor $s$ in the denominator of the vector field will introduce a numerical instability. In order to calculate thermodynamic quantities by use of a time average over the simulation data, we must assume that ergodicity is present, implying that the thermal variable $s$ must eventually sample any value arbitrarily close to zero. One might argue that a near-zero value of $s$ contributes little to the canonical distribution. But this recognition is valid only in case the system is prepared in thermal equilibrium and remains there during simulation. In practice, we rely on automatic techniques like Nosé dynamics to restore the thermal equilibrium adaptively. If an improper equilibration process is implemented before we begin the thermostatting dynamics (as is typically the case) we would expect large fluctuations of the thermal variable will be observed. As an illustration, in some of our simulations of coarse-grained materials models, thermostatting dynamics has been used to equilibrate dramatic changes of energy induced by sudden release of high-frequency phonons. The danger of sampling a near-zero value of $s$ exists in such models.

Bond et al. [5] argued that the Sundman time transformation used in the NH method destroyed the natural Hamiltonian structure of Nosé dynamics. They suggested instead a Poincaré time transformation, replacing (2.1) by

$$
H^{\mathrm{NP}}=s\left[H^{N}-E\right],
$$


where $E$ is the value of $H^{N}$ at the initial point. This is termed the Nosé-Poincaré method, and it enables the use of symplectic integration methods. It has been shown to have enhanced stability behavior in simulations [15] compared to Nosé-Hoover based methods when long time interval simulations are performed $[5,15,34]$.

\subsection{Alternative dynamical formulations}

Hoover suggested that the Nosé-Hoover formulation was unique among dynamics methods in recovering the canonical ensemble, however this uniqueness is subject to severe restrictions: there are in fact many ways to alter Nosé dynamics (besides the Nosé-Hoover and Nosé-Poincaré formulations) while still obtaining canonical sampling, among them:

- introducing a more complicated coupling among the degrees of freedom [7];

- Nosé-Hoover Chain methods based on additional thermostats [28];

- Nosé-Poincaré Chains [24];

- recursive multiple thermostats [25].

In addition, there are methods of incorporating thermostatting into only part of a simulation, or of using multiple thermostats. A number of these methods are mentioned elsewhere in this article. At this point we only discuss two methods: the idea of Bulgac and Kusnezov and a method based on the configurational rescaling.

\subsubsection{Bulgac and Kusnezov}

Bulgac and Kusnezov [7] proposed an extension of Nosé-Hoover dynamics, which clarified the essence of the thermostatting mechanism and even can be implemented for the purpose of non-Hamiltonian dynamics with thermal control. Suppose that we extend the system $(q, p)$ to a phase space $\Gamma=(q, p, \xi)$, where $\xi$ could be a vector. The basic idea for this extension is to make use of the relationship between the Liouville Equation in terms of distribution function $f$ and an extended energy function $H_{T}(\Gamma)$ :

$$
\frac{\mathrm{d} f}{\mathrm{~d} t}=-\left(\partial_{\Gamma} \cdot \dot{\Gamma}\right) f=-\frac{1}{k_{B} T} \frac{\mathrm{d} H_{T}}{\mathrm{~d} t} f,
$$

which directly results in the canonical distribution

$$
f=\mathrm{e}^{-\frac{1}{k_{B} T} H_{T}(\Gamma)} .
$$

The task then becomes to fulfill the requirement

$$
k_{B} T\left(\partial_{\Gamma} \cdot \dot{\Gamma}\right)=\frac{\mathrm{d} H_{T}}{\mathrm{~d} t}=\frac{\partial H_{T}}{\partial \Gamma} \cdot \dot{\Gamma} .
$$

It is clear that we should determine the equations of motion from the expression (2.5), and the means of formulating the dynamics is not unique. From another point of view, this process also implicitly reflects the thermal feedback mechanism of the physical dynamics. This fact can be verified by taking the ensemble average on the left side of (2.5), by the partial integration

$$
\begin{aligned}
k_{B} T\left\langle\partial_{\Gamma} \cdot \dot{\Gamma}\right\rangle & =C k_{B} T \int \partial_{\Gamma} \cdot \dot{\Gamma} \mathrm{e}^{-\frac{1}{k_{B} T} H_{T}(\Gamma)} \mathrm{d} \Gamma \\
& =-C k_{B} T \int \dot{\Gamma} \cdot \frac{\partial}{\partial \Gamma} \mathrm{e}^{-\frac{1}{k_{B} T} H_{T}(\Gamma)} \mathrm{d} \Gamma \\
& =C \int \dot{\Gamma} \cdot \frac{\partial H_{T}}{\partial \Gamma} \mathrm{e}^{-\frac{1}{k_{B} T} H_{T}(\Gamma)} \mathrm{d} \Gamma \\
& =\left\langle\dot{\Gamma} \cdot \frac{\partial H_{T}}{\partial \Gamma}\right\rangle,
\end{aligned}
$$


where $C$ is a normalization constant. So the determination process of the equations of motion naturally involves the constant temperature condition.

As an example, we consider Hamiltonian dynamics with an external field $F(q, p)$

$$
\begin{aligned}
\dot{q} & =\frac{\partial H}{\partial p}, \\
\dot{p} & =-\frac{\partial H}{\partial q}-F(q, p) .
\end{aligned}
$$

An iso-thermal dynamics can be constructed as follows:

$$
\begin{aligned}
\dot{q} & =\frac{\partial H}{\partial p} \\
\dot{p} & =-\frac{\partial H}{\partial q}-\xi F(q, p) \\
\dot{\xi} & =\left(\frac{\partial H}{\partial p} \cdot F(q, p)-K_{B} T \nabla_{p} \cdot F(q, p)\right) / \mu
\end{aligned}
$$

for which an extended energy function can be written as

$$
H_{T}=H(p, q)+\frac{\xi^{2}}{2 \mu}
$$

It is important to mention that extended dynamics obtained in this way is not any longer Hamiltonian, even though it indeed has a conserved, energy-like quantity. Thus symplectic methods are not possible within the framework of Bulgac-Kusnezov.

\subsubsection{A configurational rescaling method}

Nosé's idea was to rescale the momenta by use of an artificial thermal variable $s$, based on the natural relationship between the kinetic energy and temperature. However, we may ask the question: can we instead rescale the position by use of a (different) thermal variable $s$ ? Formally, constructing extended Hamiltonian dynamics by rescaling either momenta or positions does not substantially alter the argument for recovery of the canonical probability density of the reduced real system. A discussion of $\mathrm{NH}$ type methods in generalized form was given in [6]. As a simple illustration, consider

$$
H_{N}=\frac{p^{T} M^{-1} p}{2}+V(\tilde{q} / s)+\frac{p_{s}^{2}}{2 \mu}+g k_{B} T \ln s .
$$

By variable transformation $q=\tilde{q} / s$ and time rescaling $\mathrm{d} t=\mathrm{d} \tau / s$, we obtain

$$
\begin{aligned}
\dot{q} & =M^{-1} p-\xi q, \\
\dot{p} & =-\nabla_{q} V(q), \\
\dot{\xi} & =\left(q \cdot \nabla_{q} V(q)-g k_{B} T\right) / \mu .
\end{aligned}
$$

Note that the thermal feedback mechanism is a consequence of the classical virial theorem: $\left\langle q_{i} \nabla_{q_{j}} V\right\rangle=\delta_{i j} k_{B} T$.

In general, this position rescaling technique can be further related with the concept of configurational temperature (see e.g. $[11,20,30,32]$ ), defined by

$$
k_{B} T_{\text {conf }}=\frac{\left\langle\nabla_{q} H \cdot B(\Gamma)\right\rangle}{\left\langle\nabla_{q} \cdot B(\Gamma)\right\rangle}
$$




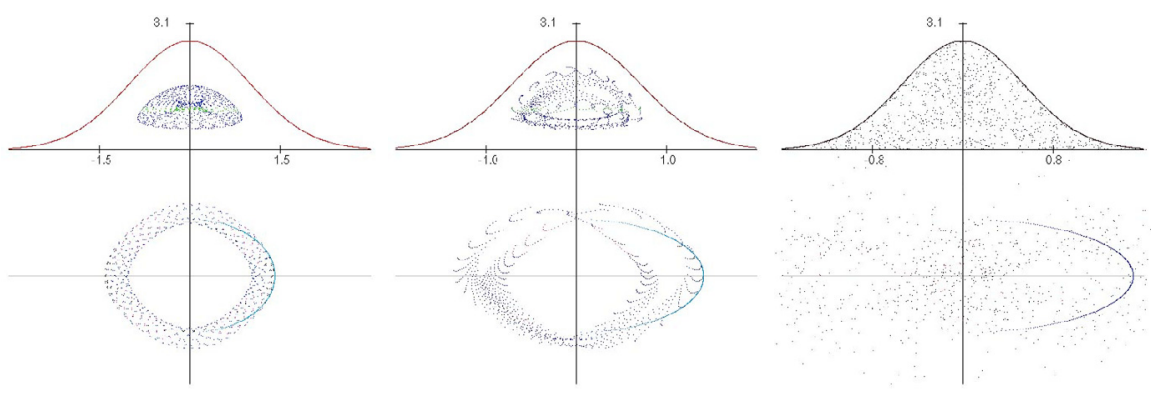

Figure 2. Illustration of the thermostatting behavior of Nosé dynamics for different choices of the thermal mass parameter. Left to right: $\mu=1, \mu=0.5$ and $\mu=0.25$. In each case the upper panel shows the $s, \pi$ phase space, while the lower panel shows evolution of the $p, q$ variables. Simulations performed at www.recursivethermostat.info.

which can be viewed as a variant of the virial theorem applied to the problems with periodic boundary conditions. A generalized Nosé-Hoover dynamics, according to Bulgac and Kusnezov's generalization, can also be constructed as

$$
\begin{aligned}
\dot{q} & =\frac{\partial H}{\partial p}-\xi \nabla_{q} B(q) \\
\dot{p} & =-\frac{\partial H}{\partial q} \\
\dot{\xi} & =\left(\nabla_{q} H \cdot B(q)-k_{B} T \nabla_{q} \cdot \nabla_{q} B(q)\right) / \mu .
\end{aligned}
$$

On the other hand, in the absence of any compelling physical or computational argument to the contrary, it is probably simpler and more reliable to use the standard approach, i.e. rescaling momenta rather than positions. Some authors have looked at rescaling the momenta in different ways $[6,36]$, but so far no obvious numerical or ergodicity advantage has been observed for such schemes.

\section{Towards A "MORE ERGodic" Nosé-Poincaré Method}

In this section, we recall the fundamental challenge for Nosé dynamics, that of obtaining ergodic sampling of the extended phase space, and then describe a method by which the Nosé-Poincaré method can be enhanced by including additional thermostatting variables, while maintaining the desirable features of the Poincaré transformation: enhanced stability, retention of Hamiltonian structure, and real-time formulation. The key point to this extension is to improve the ergodicity of the method, i.e. to introduce an artificial thermal bath that allows a more comprehensive sampling of the phase space of the system.

Let us recall the elementary example of the harmonic oscillator, which already demonstrates very well the difficulty. If the harmonic oscillator is treated using the standard Nosé scheme (in either of its time-regularized forms), the results are not very satisfying. To evaluate the quality of the sampling we obtain in this way, we plot the computed solutions in Figure 2, based on using Nosé-Poincaré and a proven symplectic integrator, for various choices of thermal mass parameter $\mu$. Here we are showing in each panel two "slices" of the dynamics: the $p, q$ phase plane and the plane of the thermostatting variables $s, \pi$.

Examine the leftmost figure which is for $\mu=1$. This certainly does not represent correct sampling! Sampling the canonical ensemble would mean sampling of the entire phase space. We could also look at the histogram of the configuration variable $q$ and we would find that it is not "close to normal". Of course the harmonic oscillator is, itself, an ergodic system, since the constant energy surface of the harmonic oscillator is always exactly covered by its trajectories. However the Nosé Hamiltonian in this case evidently is not ergodic with 
respect to the Boltzmann-Gibbs measure ${ }^{1}$. This points out the challenge if we wish to use Nosé dynamics as a sampler: we need to find a way to make it closer to an ergodic system.

\subsection{Thermal mass selection}

The most obvious thing to try is to vary the parameters used in the model and simulation. For example, we can attempt to make the numerics more accurate by decreasing the stepsize, but this does not have any substantial effect on the picture we obtain. Another thing we can try to do is to vary the thermal mass parameter $\mu$.

In Figure 2 we see a sequence of three images showing the phase portraits obtained for various choices of the thermal mass. There is clear qualitative change from one stage to the next.

The bell curves plotted in $s, \pi$ represent the solutions of the equation

$$
H^{N}(0,0, s, \pi)=0
$$

i.e. the graphs of

$$
s=\exp \left(-\frac{\pi^{2}}{2 \mu k T}\right)
$$

Since the physical energy is bounded below by zero in the case of the harmonic oscillator and $s$ is always nonnegative, it is evident that the thermostat variable phase space is contained in the bell-shaped region bounded by the indicated bell curve. On the other hand, if the system is ergodic, all points on the zero Nosé energy surface must be visited, so this means that every point in the bell shaped region must be visited. But this clearly does not happen for all choices of the thermal mass.

Optimal choice of thermal mass is related to the fundamental frequencies of the system, so in general the selection is complicated. The problem was discussed in some detail by Nosé [29] and later Martyna et al. [28], however, neither article presented estimates which were were correct for a harmonic model. In [25], the effect of the choice of thermal mass was examined more carefully in terms of the behavior of the dynamical system. It was shown to be possible to analyze systematically the harmonic oscillator and to predict the onset of chaos which carries with it the potential of correct thermalization. Yet even when this parameter is chosen optimally so that the thermal variable trajectories appear to wander throughout the bell shaped region, we still do not get good sampling. The problem is the presence of KAM tori, associated to perturbation of the integrable underlying physical model, which persist and segment the phase space. These tori correspond to islands in the thermal variable projections shown in the left hand diagram. Recently, the KAM theory was rigorously applied to Nosé dynamics by Legoll et al. [23].

This classic example illustrates the challenge for dynamical thermostats: a chaotic system is not necessarily ergodic, even in a practical sense. A certain amount of complexity is also needed, so that the tori, even if not entirely eliminated, are made very small compared to the phase space of interest. The key to carrying things forward is a new understanding of Nosé dynamics as a special case of a family of more complex thermostatting models.

\subsection{Generalizing Nosé dynamics}

Now let us discuss the extension of the basic Nosé framework for the purpose of enhancing ergodicity. The idea is to include additional degrees of freedom, coupled using the Nosé device in order to preserve the canonical measure. Many ideas along these lines have been suggested in recent years, in a non-Hamiltonian setting, including Nosé-Hoover chains (NHC) [28] and Generalized Gaussian Moment Thermostatting (GGMT) [27].

\footnotetext{
${ }^{1}$ It might be thought that perhaps the harmonic oscillator is simply "too special" and is not therefore representative of realistic systems. While typical systems that we might wish to study are generally more chaotic than the harmonic oscillator, they often include strong harmonic terms, for example the strong $\mathrm{NH}$ or $\mathrm{CH}$ bond stretches in biomolecules. It turns out that sampling of these terms creates similar difficulties, see e.g. [3]. By the same token, many models used in physics and chemistry rely on harmonic models, for example the Einstein crystal used in thermodynamic integration strategies to compute free energies of solids.
} 
In these methods, the idea is to thermostat the thermostating variable, introducing an additional variable and controlling it via an additional temperature equation. This process can be repeated, and one typically observes good ergodicity in numerical experiments with just a few additional variables.

In constructing a Hamiltonian analogue of $\mathrm{NHC}^{2}$, the important thing to remember is that if we can decouple the extended variables via transformations then we can integrate out with respect to those variables and the effect is that of introducing a constant multiple in the integrand (which will be removed in normalization). We must be careful in this process. The most natural thing would seem to be to develop a Hamiltonian version of Nosé-Hoover chains [28]. It is tempting to write down a Hamiltonian version of these chains, for example,

$$
H^{\text {chain } ; 1}=H\left(q, \frac{\tilde{p}}{s_{0}}\right)+\frac{\pi_{0}^{2}}{2 \mu s_{1}^{2}}+\frac{\pi_{1}^{2}}{2 \mu_{1}}+g k T \ln s_{0}+k T \ln s_{1} .
$$

However, it is a straightforward exercise to see that this fails due to unboundedness of the integral.

\subsubsection{Generalized baths}

In the process of asking what device is needed to fix the Hamiltonian $H^{\text {chain }}$, we can also ask the more general question: "Which Hamiltonian extensions will reduce to canonical sampling?" In this way we are led to consider Hamiltonians of a very general form

$$
H^{\mathrm{GN}}=H\left(q, \tilde{p} / \Pi s_{\alpha}\right)+\bar{H}\left(s_{0}, s_{2}, \ldots, s_{m}, \pi_{1}, \pi_{2}, \ldots, \pi_{m}\right) .
$$

Here the terms $\left\{s_{\alpha}\right\}$ represent some subset of the thermostatting variables. The requirement for sampling is that

$$
\iint f\left(q, \tilde{p} / \Pi s_{\alpha}\right) \delta\left[H^{\mathrm{GN}}-E\right] \mathrm{d} s \mathrm{~d} \pi \mathrm{d} \tilde{p}=f(q, p) \exp (-(1 / k T) H(q, p)) \mathrm{d} p .
$$

It is important that all momenta are modified by an identical rescaling so that we may introduce an appropriate Poincaré transformation to correct the scaling of time in the differential equations.

What is remarkable is how weak the restriction is. We can effectively bathe any given system in any other system. If we choose, for example,

$$
H^{\mathrm{GN}}=H\left(q, p / s_{0}\right)+a\left(s_{1}, s_{2}, \ldots, s_{m}\right) \pi_{0}^{2}+g k T \ln s_{0}+G\left(s_{1}, s_{2}, \ldots, s_{m}, \pi_{1}, \pi_{2}, \ldots, \pi_{m}\right),
$$

where $G$ is an arbitrary Hamiltonian whose key properties are that it be bounded below and have bounded exponential integral:

$$
\iint \exp \left(-\frac{1}{k T} G\right) \mathrm{d} s \mathrm{~d} \pi<\infty
$$

In [2], several such baths were proposed, including (i) a set of independent harmonic oscillators, "vertex coupling" and (ii) a "nearly ergodic" system of firm spheres in a box. With a suitable choice of parameters, both of these simple models were able to thermostat the harmonic oscillator (i.e. we plotted and compared the position distributions and found them satisfyingly normal). But they are not the most efficient schemes.

\subsubsection{Nosé-Poincaré chains and recursive multiple thermostats}

Using ideas from [2], it is possible to make the Hamiltonian chains (3.1) work [24]. The key correction needed is to add a "regularizing" term in the Hamiltonian, or several regularizing functions of the thermostatting variables $s_{i}, i \geq 1$. If these functions are bounded below with suitably bounded exponential integral, then the integration argument goes through and a canonical sampling argument exists in this case.

We can make these thermostatting chains as complicated as we like by extending them with more variables. For the right choice of parameters, Nosé-Poincaré chains can achieve thermostatting in some types of models: if we use 3 or 4 augmenting variables and choose carefully the thermal masses $\mu_{1}, \mu_{2}, \ldots$, then we can get an

\footnotetext{
${ }^{2}$ Due to the means of construction, GGMT does not appear to have a natural Hamiltonian analogue.
} 
accurate recovery of canonical sampling from these chains. But there is an even more versatile and powerful class of methods.

In what sense? If we have two methods that generate canonical sampling, how do we say which is better? The question is important but not easy to answer precisely, i.e. in a rigorous way. There are no criteria that one can write down and use to check whether a given method is a good one. One can only give numerical comparisons in terms of particular model problems. And different model problems can give very different results! The task of evaluating methods is made even more challengin by the fact that ultimately our intention is to mimic a stochastic process; the numerical results are fundamentally subject to random error and it is very difficult to assess the behavior of methods using traditional techniques of (deterministic) numerical analysis. For example, one trajectory out of 10 with a given method may result in an extremely large energy error, while all others are much smaller. Numerical results may even be dependent on the conformations visited by a molecule; thus a less ergodic simulation can be more numerically stable than a fully ergodic one. Probably it will be necessary to undertake a large scale simulation with a full sampling of initial data in order to reach completely satisfactory conclusions regarding method comparison.

In our experience, to get methods that perform well, we need to introduce a more complicated interaction between the bath and the physical variables. The result is a technique called Recursive Multiple Thermostatting, which we describe via the Hamiltonian

$$
\mathcal{H}_{N P}^{R M T}=s_{1} \ldots s_{r}\left(\mathcal{H}_{\text {Nosé }}^{R M T}-\mathcal{H}_{0}\right)
$$

where

$$
\begin{aligned}
\mathcal{H}_{\text {Nosé }}^{R M T}= & \frac{p^{T} M^{-1} p}{2 s_{1}^{2} \ldots s_{r}^{2}}+V(q)+\sum_{k=1}^{r-1} \frac{\pi_{k}^{2}}{2 \mu_{k} s_{k+1}^{2} \ldots s_{r}^{2}}+\frac{\pi_{r}^{2}}{2 \mu_{r}} \\
& +g k_{B} T \ln s_{1}+\sum_{k=2}^{r}\left((g+k-1) k_{B} T \ln s_{k}+\frac{\left(1-s_{k}\right)^{2}}{2 C_{k}}\right) .
\end{aligned}
$$

The theory and practical treatment (e.g. discretization) of this model are not completely trivial and are discussed in more detail in $[3,25]$. Extensive computer experimentation with these formulations has shown that the correct parameters (thermostat masses) in the RMT formulation are important to obtain good results. This observation has very important advantages for the development of practical numerical approaches. Numerical experiments in [8] suggest that there is still some work to do on the implementation of Hamiltonian thermostatting chains before they will match the high numerical performance standard set by the Nosé-Poincaré method.

\section{Projection And thermostatting}

In this section, we describe a procedure to combine projection and thermostatting techniques into a single coherent framework. This section is built on our recent work [21]. The determination of a projection operator is largely dependent on the modelling perspective, specifically whether the goal is sampling or dynamics in a constant temperature environment.

For sampling, we would like to choose the projection operator over some critical manifold on which the system needs to be specially thermostatted to enhance the ergodicity while staying at or near the specified target temperature. In [21], we have discussed the problem associated to the Boltzmann [4] and Jeans [18,19] conjecture: the time scale for a set of fast vibrational modes to share energy grows exponentially with the vibrational frequency, i.e. high frequencies become in essence decoupled and thus frozen. On realistic timescales, the detailed evolution of adiabatic vibrational components can be viewed as having no direct impact on the slow dynamics, and energy exchanges with fast vibrational degrees of freedom are too small to contribute to the thermal equilibration of the whole system. 


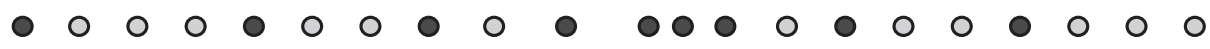 \\ - Representative Atom \\ - Slave Atom}

Figure 3. Coarse-grained geometry of a 1D chain with representative atoms (dark) and slave atoms (light).

On the other hand, for studying the dynamics of a multiscale system at finite temperature, we should construct the projection over the degrees of freedom that are of no practical dynamical interest for the physical or chemical purposes of simulation. In some cases, we may need to determine the projection based on both of these perspectives. In the following, we will present some applications to explain the use of projection and thermostatting in different contexts.

\subsection{Simulation of coarse-grained models using partial thermostats}

One of the motivations of projective thermostatting is to retain the dynamics of interest in such a way that the details of evolution are shielded from effects of artificial thermostatting.

As an example, we could consider a solid material containing a defect (such as a dislocation or crack). In order to keep the constant temperature for the system, a heat bath has to be connected to the material. We can imagine that the heat bath will be only connected to the boundary atoms of material rather than the defect itself. The defect will interact directly with the surrounding atoms and thereby exchange heat. More importantly, as we know, the dynamical behavior of the defect is crucial for us to test the macroscopic response and performance of the material. We therefore aim to retain an accurate model for the true dynamics of the defect.

For the purposes of illustration, we have recently studied in [14] an atomic Lennard-Jones chain by use of coarse-grained molecular dynamics

$$
H_{C G}(q, p)=\frac{p^{T} m^{-1} p}{2}+\sum_{i=1}^{n-1} n_{i} \phi\left(r_{i}\right)+\frac{1}{2} k_{B} T \sum_{i=1}^{n-1}\left(n_{i}-1\right) \ln \left(\left.\frac{\partial^{2} \phi}{\partial r^{2}}\right|_{r=r_{i}}\right)
$$

where $\phi(r)=4 \epsilon\left(\left(\frac{\sigma}{r}\right)^{12}-\left(\frac{\sigma}{r}\right)^{6}\right)$ and $r_{i}=\left(q_{i+1}-q_{i}\right) / n_{i}$ is the mean interatomic spacing in element $i$.

In the defect region, the atomic structure is retained to reflect the detailed nonlinear dynamical behavior, while a coarse-grained structure is obtained for the field far from the defect. An extended Hamiltonian that describes this type of formulation would be as follows:

$$
H_{P T}=\frac{p_{a}^{T} M_{a}^{-1} p_{a}}{2 s^{2}}+\frac{p_{b}^{T} M_{b}^{-1} p_{b}}{2}+\frac{\pi^{2}}{2 \mu}+V_{C G}(q, T)+g k_{B} T \ln s
$$

where $p_{a}$ and $p_{b}$ represent momenta corresponding to the sets of thermostatted and unthermostatted components, respectively, with corresponding mass matrices $M_{a}$ and $M_{b}$; the two sets of variables are coupled in the potential energy term. In practice, the ergodicity is inadequate using this type of approach, particularly as the coarse-grained part of the system is typically close to linear. To correct the situation, it is suggested to use an RMT thermostat in place of the Nosé type thermostat. In our model, this thermostat is only applied to the coarse-grained region, while Newtonian interaction should be retained between the defect and surrounding atoms.

There is an additional problem if we wish to incorporate a time-transformation at the level of the Hamiltonian (Poincaré transformation) to resolve numerical stability issues. The difficulty is that the time rescaling would 

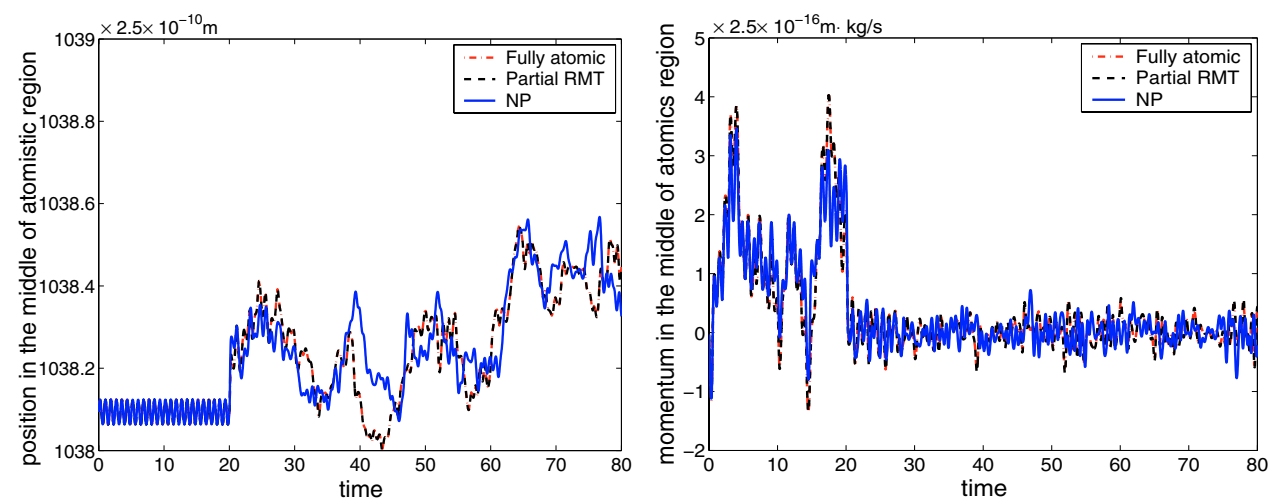

Figure 4. Momentum and position in the middle of AR, for fully (NP) and partially (PTMD) thermostatted simulations, compared with a constant energy fully atomistic model.

then affect all variables of the system. Under the assumption of scale separation in the physical coarse-grained model, the time-transformation would introduce a mixing of all scales and corrupt the real-time correspondence in the unthermostatted part. The approach given in [21] is an intermediate compromise which allows us to treat all variables of the system in real-time. While giving up the Hamiltonian structure, it retains time-reversibility and volume preservation in phase space. At the same time, it has the property that the system that results is in the form of a pair of coupled Hamiltonian systems each subject to perturbation which, under the assumption of adiabatic separation, will remain adiabatic. Rather than writing out all the details of this combined scheme for the RMT form of the thermostat, we simply describe the PTMD equations corresponding to $H_{P T}$ :

$$
\begin{aligned}
\frac{\mathrm{d}}{\mathrm{d} t} q_{b} & =M_{b}^{-1} p_{b} \\
\frac{\mathrm{d}}{\mathrm{d} t} p_{b} & =-\nabla_{q_{b}} V_{C G} \\
\frac{\mathrm{d}}{\mathrm{d} t} p_{a} & =\frac{1}{s} M_{a}^{-1} p_{a} \\
\frac{\mathrm{d}}{\mathrm{d} t} p_{b} & =-s \nabla_{q_{b}} V_{C G} \\
\frac{\mathrm{d}}{\mathrm{d} t} s & =s \pi / \mu \\
\frac{\mathrm{d}}{\mathrm{d} t} \pi & =p_{a}^{T} M_{a}^{-1} p_{a}-g k_{B} T-\Delta H_{P T} .
\end{aligned}
$$

The extension to RMT thermostatting is straightforward.

In practice, a still more complicated formulation is used to break down the energy locking in the 1-D chain model, as described in [14]. Between the atomistic region and a heavily coarse-grained extended region, an interface region is inserted. To this interface region we apply yet another layer of thermostatting using the PTMD idea. A method based on this was applied to the coarse-grained 1-D chain model. The chain used consisted of 1250 atoms, with only the 200 central atoms in the atomistic region (AR). A 9-fold coarse grained (CG) region was used with 48 representative atoms in the outermost part on each end of the chain, and then the intermediate interface (IF) region on each side consisted of $7-5-3-1$-fold coarse graining with $5,5,5$, and 18 representative atoms in each part. The system was modelled with an initial condition designed to study sampling and phonon absorption. We can see good dynamical and sampling behavior in the Figures 4 and 5; figures similar to Figure 5 first appeared in [14], where many more details can be found. 

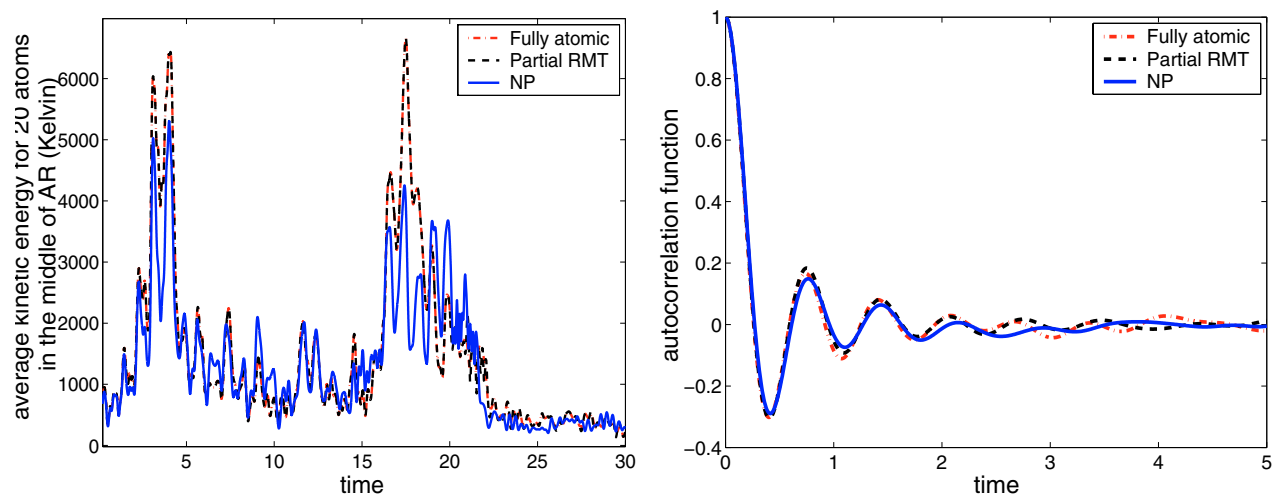

FiguRE 5. Kinetic energy fluctuations for the inner 20 atoms in the AR, for fully (NP) and partially (PTMD) thermostatted simulations, compared with a constant energy fully atomistic model (left); velocity autocorrelation functions for PTMD and atomistic models: applying strong thermostatting to IF and CG regions has not severely distorted the velocity autocorrelation functions inside the AR (right).

\subsection{Projective isothermal-isobaric dynamics}

We would like to describe how to implement both volume control of selective coordinates and, simultaneously, thermal control of selective momenta. In order for the discussion to be as general as possible, we set up the projection over the position and momentum as

$$
\begin{aligned}
p & =\mathcal{P}_{T} p+\mathcal{Q}_{T} p \\
q & =\mathcal{P}_{P} q+\mathcal{Q}_{P} q
\end{aligned}
$$

and $\mathcal{P}_{T}$ is defined as

$$
\mathcal{P}_{T}=\chi^{T}\left(\chi M^{-1} \chi^{T}\right)^{-1} \chi M^{-1}
$$

where $\chi=\nabla_{q} r(q)$ and $r(q)$ is chosen for some favorable purpose.

The projective thermostatting and barostatting dynamics (PTBD) can be formulated with the Hamiltonian:

$$
\begin{aligned}
H= & \frac{p^{T} \mathcal{A}_{V}^{T} \mathcal{P}_{T}^{T} M^{-1} \mathcal{P}_{T} \mathcal{A}_{V} p}{2 s^{2} V^{2 / 3}}+\frac{p^{T} \mathcal{A}_{V}^{T} \mathcal{Q}_{T}^{T} M^{-1} \mathcal{Q}_{T} \mathcal{A}_{V} p}{2 V^{2 / 3}}+U\left(V^{1 / 3} \mathcal{A}_{V}^{-1} q\right) \\
& +\frac{p_{s}^{2}}{2 \mu}+g k_{B} T \ln s+\frac{p_{V}^{2}}{2 W}+P_{e x} V,
\end{aligned}
$$

where

$$
\begin{aligned}
\mathcal{A}_{V} & =\mathcal{P}_{P}+V^{1 / 3} \mathcal{Q}_{P}, \\
\mathcal{A}_{V}^{-1} & =\mathcal{P}_{P}+V^{-1 / 3} \mathcal{Q}_{P} .
\end{aligned}
$$

We can prove the following theorem:

Theorem 4.1. The PTBD subject to the Hamiltonian (4.5) samples the Gibbs distribution, i.e.

$$
\rho\left(p^{\prime}, q^{\prime}, V\right)=\exp ^{-\frac{1}{k_{B} T}\left(H_{0}\left(p^{\prime}, q^{\prime}\right)+P_{e x} V\right)},
$$

where $H_{0}\left(q^{\prime}, p^{\prime}\right)=\frac{p^{\prime T} M^{-1} p^{\prime}}{2}+U\left(q^{\prime}\right)$ and $q^{\prime}, p^{\prime}$ represent the real variables. 
Regarding the time rescaling issue for Nosé type dynamics, we transform the Hamiltonian (4.5) into the form:

$$
H_{P T}=s\left(H-H_{0}\right) \triangleq s \triangle H,
$$

for which the equations of motion to PTBD are

$$
\begin{aligned}
\dot{q} & =\frac{\mathcal{A}_{V}^{T} \mathcal{P}_{T}^{T} M^{-1} \mathcal{P}_{T} \mathcal{A}_{V} p}{s V^{2 / 3}}+s \frac{\mathcal{A}_{V}^{T} \mathcal{Q}_{T}^{T} M^{-1} \mathcal{Q}_{T} \mathcal{A}_{V} p}{V^{2 / 3}}, \\
\dot{p} & =-s V^{1 / 3} \mathcal{A}_{V}^{-1} \nabla_{q} U\left(V^{1 / 3} \mathcal{A}_{V}^{-1} q\right), \\
\dot{s} & =s \frac{p_{s}}{\mu} \\
\dot{V} & =s \frac{p_{V}}{W} \\
\dot{p}_{s} & =\left(\frac{p^{T} \mathcal{A}_{V}^{T} \mathcal{P}_{T}^{T} M^{-1} \mathcal{P}_{T} \mathcal{A}_{V} p}{s^{2} V^{2 / 3}}-g k_{B} T\right)-\triangle H, \\
\dot{p}_{V} & =s\left(P_{\text {int }}-P_{e x}\right),
\end{aligned}
$$

where the internal pressure $P_{\text {int }}$ is defined by

$$
P_{\text {int }}=\frac{1}{3 V}\left(\frac{p^{T} \mathcal{A}_{V}^{T} \mathcal{P}_{T}^{T} M^{-1} \mathcal{P}_{T} \mathcal{A}_{V} p}{s^{2} V^{2 / 3}}+\frac{p^{T} \mathcal{A}_{V}^{T} \mathcal{Q}_{T}^{T} M^{-1} \mathcal{Q}_{T} \mathcal{A}_{V} p}{V^{2 / 3}}-\frac{\partial U}{\partial q} q\right)
$$

In general, PTMD is a non-separable Hamiltonian dynamics since the projection matrices $\mathcal{P}_{T}$ and $\mathcal{P}_{P}$ are dependent on the position. Thus a generalized symplectic Verlet method is preferred to the numerical integration.

In practice, we may be interested in the simplified formulations in which the pressure and temperature controls are applied in special ways.

Corollary 1. Taking the same projection operator for the position and momentum, i.e.

$$
\mathcal{P}_{T}=\mathcal{P}_{P}
$$

the original Hamiltonian (4.5) becomes

$$
H=\frac{p^{T} \mathcal{P}_{P}^{T} M^{-1} \mathcal{P}_{P} p}{2 s^{2} V^{2 / 3}}+\frac{p^{T} \mathcal{Q}_{P}^{T} M^{-1} \mathcal{Q}_{P} p}{2}+U\left(V^{1 / 3} \mathcal{A}_{V}^{-1} q\right)+\frac{p_{s}^{2}}{2 \mu}+g k_{B} T \ln s+\frac{p_{V}^{2}}{2 W}+P_{e x} V .
$$

If we further choose the trivial projection operator

$$
\mathcal{P}_{T}=\mathcal{P}_{P}=\left(\begin{array}{ll}
I & 0 \\
0 & 0
\end{array}\right),
$$

the system describes the case that only a subset of the degrees of freedom are connected with heat and pressure baths, while the other part retain Newtonian interactions.

This idea could be naturally applicable to the realistic simulation, where an intrinsic multiscale structure is present and the system is implemented at constant temperature and pressure. The pressure and temperature control will be connected only with one part of variables, therefore the multiscale structure is preserved and the dynamics for the other subset of variables is shielded from the artificial temperature and pressure rescaling effect. The corresponding Hamiltonian can be written as

$$
H_{\text {part }}=\frac{p_{1}^{T} M^{-1} p_{1}}{2 s^{2} V^{2 / 3}}+\frac{p_{2}^{T} M^{-1} p_{2}}{2}+U\left(V^{1 / 3} q_{1}, q_{2}\right)+\frac{p_{s}^{2}}{2 \mu}+g k_{B} T \ln s+\frac{p_{V}^{2}}{2 W}+P_{e x} V .
$$


It is noted that a partial thermostatting and barostatting dynamics can be obtained in a similar way with that of partial thermostatting dynamics in [21]. We can trivially write down the equations:

$$
\begin{aligned}
\dot{q}_{1} & =\frac{M_{1}^{-1} p_{1}}{s V^{2 / 3}} \\
\dot{p}_{1} & =-s V^{1 / 3} \nabla_{q_{1}} U\left(V^{1 / 3} q_{1}, q_{2}\right), \\
\dot{s} & =s \frac{p_{s}}{\mu} \\
\dot{p}_{s} & =\frac{p_{1}^{T} M_{1}^{-1} p_{1}}{s^{2} V^{2 / 3}}-g k_{B} T-\triangle H \\
\dot{q}_{2} & =M_{2}^{-1} p_{2}, \\
\dot{p}_{2} & =-\nabla_{q_{2}} U\left(V^{1 / 3} q_{1}, q_{2}\right),
\end{aligned}
$$

where $\triangle H=H_{\text {part }}-H_{0}$. The numerical method can be constructed by the appropriate composition of numerical integrators solving two subsystems. An explicit generalized Verlet method for the subsystem consisting of $q_{1}, p_{1}$ can be constructed referring to [34]. Since the velocity Verlet method can be applied to the subsystem consisting of $q_{2}, p_{2}$, the overall integrator is explicit. If two subsystem are of disparate time scales, a generalized reversible averaging method [21] can be applied.

\section{Separate thermostats and multiple temperature Simulation}

The previous section discussed how we could introduce additional thermostats in order to enhance the ergodicity of the system. An even more challenging question is: can we construct a Hamiltonian formulation with a dual-temperature control for different parts of the system?

As a first step, one could think of having separate controls adjusted to the dynamical features in different parts of the model and aimed at realizing the canonical sampling at a given target temperature. Alternatively, and more ambitiously, one could ask what would happen if we relax the requirement that the temperatures in different parts of the model be equal, while maintaining the ensemble averages for one part of system. Let us first emphasize that it is essentially impossible to construct such a Hamiltonian formulation within the Nosé thermostatting framework. The natural formulation for a two-temperature control would have the following form:

$$
\begin{aligned}
H_{\text {Nosé }}= & s_{1} s_{2}\left(\frac{p_{1}^{T} M_{1}^{-1} p_{1}}{2 s_{1}^{2}}+\frac{p_{2}^{T} M_{2}^{-1} p_{2}}{2 s_{2}^{2}}+V\left(q_{1}, q_{2}\right)\right. \\
& \left.+\frac{\pi_{1}^{2}}{2 \mu_{1}}+\frac{\pi_{2}^{2}}{2 \mu_{2}}+g_{1} k_{B} T_{1} \ln s_{1}+g_{2} k_{B} T_{2} \ln s_{2}+\phi\left(s_{1}\right)-E\right) .
\end{aligned}
$$

We would like to keep the ensemble average (at temperature $T_{2}$ ) of the subsystem in terms of $q_{2}$ and $p_{2}$, and consider what form of regularized term $\phi\left(s_{1}\right)$ should be used. Let us check the partition function:

$$
Z=\int \mathrm{d} q \mathrm{~d} p \mathrm{~d} s_{1} \mathrm{~d} s_{2} \mathrm{~d} \pi_{1} \mathrm{~d} \pi_{2} \delta\left(s_{1} s_{2}\left(H_{N o s e ́}-E\right)\right) .
$$

By similar algebraic manipulation to that used in Nosé's sampling argument, the partition function can be rewritten as

$$
Z=C \int \mathrm{d} \bar{q}_{1} \mathrm{~d} \bar{q}_{2} \mathrm{~d} \bar{p}_{2} \mathrm{~d} s_{1} s_{1}^{N_{1}-1} \mathrm{e}^{-\frac{1}{k_{B} T_{2}}\left(V\left(\bar{q}_{1}, \bar{q}_{2}\right)+\frac{\bar{p}_{2}^{T} M_{2}^{-1} \bar{p}_{2}}{2}+g_{1} k_{B} T_{1} \ln s_{1}+\phi\left(s_{1}\right)\right)}
$$


where $\mathrm{C}$ is a constant and $\bar{q}, \bar{p}$ correspond to the real variables. We next must check if the integrand has bounded integral with respect to $s_{1}$. This integrand can be written as

$$
s_{1}^{N_{1}-1} \mathrm{e}^{-\frac{1}{k_{B} T_{2}}\left(g_{1} k_{B} T_{1} \ln s_{1}+\phi\left(s_{1}\right)\right)}=s_{1}^{N_{1}-\frac{T_{1}}{T_{2}} g_{1}-1} \mathrm{e}^{-\frac{\phi\left(s_{1}\right)}{k_{B} T_{2}}} .
$$

Lemma 5.1. Assuming that (5.4) has bounded integral with respect to $s_{1}$, then we have

$$
\begin{aligned}
\left\langle\frac{p_{1}^{T} M_{1}^{-1} p_{1}}{s_{1}^{2}}\right\rangle & =N_{1} k_{B} T_{2}, \\
\left\langle\frac{p_{2}^{T} M_{2}^{-1} p_{2}}{s_{2}^{2}}\right\rangle & =N_{2} k_{B} T_{2}, \\
\left\langle s_{1} \nabla_{s_{1}} \phi\left(s_{1}\right)\right\rangle & =N_{1} k_{B} T_{2}-g_{1} k_{B} T_{1} .
\end{aligned}
$$

We see that the averages of kinetic energy associated with different projective modes ultimately corresponds to the same temperature $T_{2}$, which contradicts the aim to control different modes with different temperatures. The regularized term $\phi\left(s_{1}\right)$ provides the additional contribution which has the effect of eliminating the temperature difference.

In general, we can consider the issue in a more abstract sense. Different-temperature control means that the system is inevitably maintained in a pre-equilibrium condition, with an effective temperature gradient present in the dynamics. This directly contradicts the idea that subdynamics associated to a subset of phase variables will sample a canonical distribution, assuming that there exists a strong interaction between the different variables. The heat flow will destroy the equilibrium canonical distribution of the subsystem. Nosé, Nosé-Poincaré, and Nosé-Hoover dynamics are incapable of sampling an ergodic (strongly mixing) system at two distinct temperatures.

The only possible exception, albeit an intriguing one, arises if there is a strong adiabatic decoupling between different subsystems. This would imply a weak interaction between different degrees of freedom, inducing a very slow process of heat transfer between the subsystems. The subsystems will persist in separate (metastable) pre-equilibrium conditions, possibly for a very long time. The weak dependence of the different parts finally contributes to the derivation of a potential of mean force, so that we can treat the subsystems in a sequential way. A similar technique is used in the setting of Car-Parinello dynamics [9], and a related approach is used for free energy profile calculation [31] in an on-the-fly numerical averaging process.

To make all this concrete, we consider a system with two boundary regions connected to hot and cold heat baths respectively, as proposed in [17]. The variables in boundary regions 1 and 2 are denoted by $q_{B_{1}}, q_{B_{2}}$, with similar notation for the corresponding momenta. A Hamiltonian formulation is then suggested:

$$
\begin{aligned}
H_{N P T}= & \frac{p_{B_{1}}^{T} M_{B_{1}}^{-1} p_{B_{1}}}{2 s_{1}^{2}}+\frac{p_{B_{2}}^{T} M_{B_{2}}^{-1} p_{B_{2}}}{2 s_{2}^{2}}+\frac{p^{T} M^{-1} p}{2}+V\left(q_{B_{1}}, q_{B_{2}}, q\right) \\
& +\frac{\pi_{1}^{2}}{2 \mu_{1}}+\frac{\pi_{2}^{2}}{2 \mu_{2}}+g_{1} k_{B} T_{1} \ln s_{1}+g_{2} k_{B} T_{2} \ln s_{2} .
\end{aligned}
$$

In order to resolve the numerical instability induced by the virtual time scale, we could simply take the following transformed Hamiltonian

$$
H_{N P P T}=s_{1} s_{2}\left(H_{N P T}-H_{0}\right) \text {. }
$$

However, we notice that the time scale for each thermostatting subsystem actually differs from the real time scale by a factor of $s_{2}$ or $s_{1}$. Even worse, the introduced coupling between thermal variables and $q, p$ would be expected to corrupt the dynamics between two heat baths and lead to undesirable artifacts in the modelling. The solution is of course to use a partial thermostatting method which is designed for the two-temperature formulation. 


\subsection{Partial thermostatting dynamics with multi-temperature control}

We suggest the following dynamics

$$
\begin{aligned}
\dot{q}_{B_{1}} & =\frac{M_{B_{1}}^{-1} p_{B_{1}}}{s_{1}}, \\
\dot{p}_{B_{1}} & =-s_{1} \nabla_{q_{B_{1}}} V \\
\dot{s}_{1} & =s_{1} \frac{\pi_{1}}{\mu_{1}} \\
\dot{\pi}_{1} & =\frac{p_{B_{1}}^{T} M_{B_{1}}^{-1} p_{B_{1}}}{2 s_{1}^{2}}-g_{1} k_{B} T_{1}-\triangle H \\
\dot{q}_{B_{2}} & =\frac{M_{B_{2}}^{-1} p_{B_{2}}}{s_{2}}, \\
\dot{p}_{B_{2}} & =-s_{2} \nabla_{q_{B_{2}}} V \\
\dot{s}_{2} & =s_{2} \frac{\pi_{2}}{\mu_{2}}, \\
\dot{\pi}_{2} & =\frac{p_{B_{2}}^{T} M_{B_{2}}^{-1} p_{B_{2}}}{2 s_{2}^{2}}-g_{2} k_{B} T_{2}-\triangle H, \\
\dot{q} & =M^{-1} p, \\
\dot{p} & =-\nabla_{q} V,
\end{aligned}
$$

where

$$
\triangle H=H_{N P T}-H_{0} .
$$

Each of the three subsystems evolves in real time. The effect is of a pair of Nosé-Poincaré-like systems coupled to Newtonian dynamics. This dynamics has the same geometric properties as PTMD in [21], summarized in the following theorem:

Theorem 5.2. The dynamics (5.9)-(5.18) is time-reversible and preserves the phase volume and the first integral

$$
s_{1} s_{2} \triangle H .
$$

The proof is elementary. Numerical methods can be constructed to preserve the time-reversal symmetry and (extended) phase space volume. The formulation is similar to the way that two temperatures would be implemented using Nosé-Hoover methods, but it is thought that the additional correction terms in (5.12) and (5.16) will confer additional stability.

We do not present detailed numerical comparisons with alternatives at this time, but we have studied this generalized partial thermostatting technique for a simple application which is similar to the example of [17]. A monatomic Lennard-Jones chain was set up as a combination of three parts: hot bath (100 atoms), cold bath (500 atoms) and the dynamics in between (100 atoms), which is typically a heat conduction test in the context of non-equilibrium statistical mechanics. All of the atoms in the chain are initially set at their equilibrium points. The temperatures (in reduced unit) are initially set up as $1(7000 \mathrm{~K})$ for the hot bath, 0.1 (700 K) for the cold bath and $0(0 \mathrm{~K})$ for the dynamics in between. In Figure 6 , we can see the fast equilibration of the non-thermostatted system, while the pair of heat baths render a temperature gradient for the dynamics in between. The provided experiment is only proof-of-concept for the new multiple thermostat technique; much further experiment is needed to assess the possible benefit of this approach.

Remark. We have described a non-equilibrium heat conduction problem. This multi-temperature dynamics, with a regularizing term incorporated, can also be used to provide local thermostatting in different regions for equilibrium problems. 

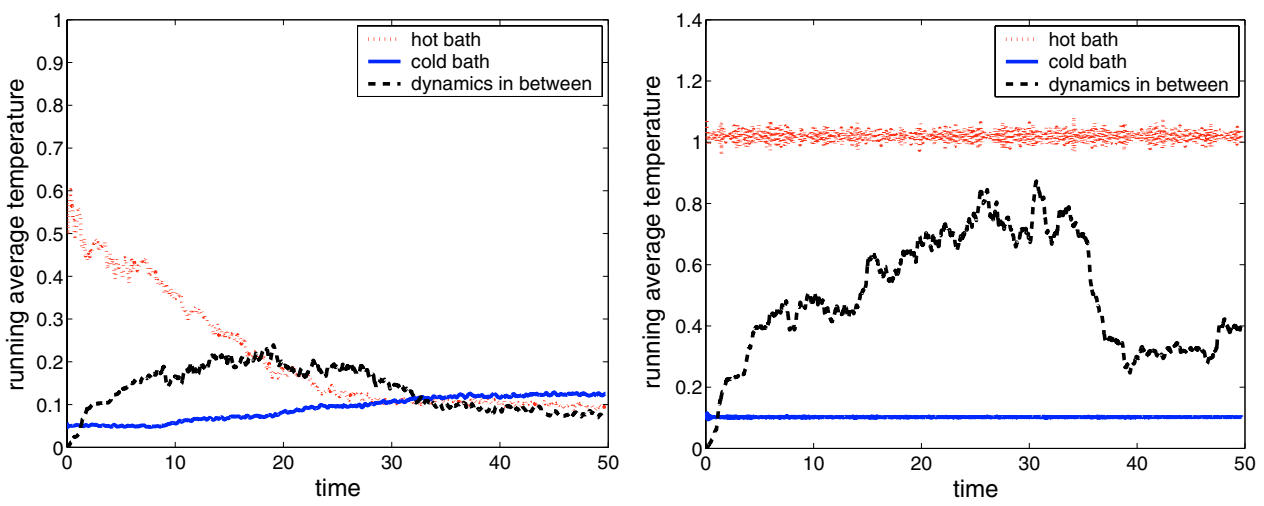

Figure 6. Two temperature simulation in a LJ chain: without thermostatting (left) and thermostatting with two different baths (right).

\section{Conclusion}

In this paper, we have reviewed and developed deterministic thermostatting techniques based on generalizations of Nosé's Hamiltonian formulation. Projective thermostatting dynamics techniques have been presented in a unified framework incorporating flexible treatment of thermostatting in combination with practical sampling or dynamic concerns and multiscale modelling perspectives. Partial thermostatting techniques, recovering the real time scale of the whole system, have been described to treat the problem of thermostatting different degrees of freedom with the same or different temperatures. In addition, a generalized projective isothermal-isobaric dynamic formulations has been introduced.

Acknowledgements. The authors thank W. Hoover for comments on a draft of the paper. This work was initiated while Dr Zia was supported by the Engineering and Physical Science Research Council (UK), grant number GR/R03259/01.

\section{REFERENCES}

[1] H.C. Andersen, Molecular dynamics simulations at constant pressure and/or temperature. J. Chem. Phys. 72 (1980) 23842393.

[2] E.J. Barth, B.B. Laird and B.J. Leimkuhler, Generating generalized distributions from dynamical simulation. J. Chem. Phys. 118 (2003) 5759-5768.

[3] E. Barth, B. Leimkuhler and C. Sweet, Approach to thermal equilibrium in biomolecular simulation, in New Algorithms for Macromolecular Simulation, B. Leimkuhler, C. Chipot, R. Elber, A. Laaksonen, A. Mark, T. Schlick, C. Schütte and R. Skeel Eds., Springer Lecture Notes in Computational Science and Engineering 49 (2006).

[4] L. Boltzmann, On certain questions of the theory of gases. Nature 51 (1895) 413-415.

[5] S.D. Bond, B.J. Leimkuhler and B.B. Laird, The Nosé-Poincaré method for constant temperature molecular dynamics. J. Comput. Phys. 151 (1999) 114-134.

[6] A.C. Branka and K.W. Wojciechowski, Generalization of Nosé and Nosé-Hoover isothermal dynamics. Phys. Rev. E 62 (2000) 3281-3292.

[7] A. Bulgac and D. Kusnezov, Canonical ensemble averages from pseudomicrocanonical dynamics. Phys. Rev. A 42 (1990) $5045-5048$

[8] E. Cancés, F. Legoll and G. Stoltz, Theoretical and numerical comparison of some sampling methods for molecular dynamics. ESAIM: M2AN (to appear).

[9] R. Car and M. Parinello, Unified approach for molecular dynamics and density function theory. Phys. Rev. Lett. 55 (1985) $2471-2475$

[10] C. Chipot, Free energy calculations in biological systems: how useful are they in practice, in New Algorithms for Macromolecular Simulation, B. Leimkuhler, C. Chipot, R. Elber, A. Laaksonen, A. Mark, T. Schlick, C. Schütte and R. Skeel Eds., Springer Lecture Notes in Computational Science and Engineering 49 (2006). 
[11] J. Delhommelle, Correspondence between configurational temperature and molecular kinetic temperature thermostats. $J$. Chem. Phys. 117 (2002) 6016-602.

[12] E.G. Flekkøy and P.V. Coveney, From molecular dynamics to dissipative particle dynamics. Phys. Rev. Lett. 83 (1999) 17751778.

[13] D. Frenkel and B. Smith, Understanding Molecular Simulation. Academic, London (1996).

[14] S.P.A. Gill, Z. Jia, B. Leimkuhler and A.C.F. Cocks, Rapid thermal equilibration in coarse-grained molecular dynamics. Phys. Rev. B 73 (2006) 184304.

[15] E. Hernandez, Metric-tensor flexible-cell for isothermal-isobaric molecular dynamics simulation. J. Chem. Phys. 115 (2001) $10282-10290$.

[16] W.G. Hoover, Canonical dynamics: Equilibrium phase-space distributions. Phys. Rev. A 31 (1985) 1695-1697.

[17] W.G. Hoover, K. Aoki, C.G. Hoover and S.V. De Groot, Time reversible deterministic thermostats. Physica D (2004) 253-267.

[18] J.H. Jeans, On the vibrations set up in molecules by collisions. Phil. Mag. 6 (1903) 279-286.

[19] J.H. Jeans, On the partition of energy between matter and ether. Phil. Mag. 10 (1905) 91-97.

[20] O.G. Jepps, G. Ayton and D.J. Evans, Microscopic expressions for the thermodynamic temperature. Phys. Rev. E, 62 (2000) $4757-4763$

[21] Z. Jia and B.J. Leimkuhler, A projective thermostatting dynamics technique. Multiscale Model. Simul. 4 (2005) 563-583.

[22] D. Kusnezov, Diffusive aspects of global demons. Phys. Lett. A 166 (1992) 315-320.

[23] F. Legoll, M. Luskin and R. Moeckel, Non-ergodicity of the Nosé-Hoover thermostatted harmonic oscillator, arXiv preprint (November 2005, math.DS/0511178). ARMA (to appear).

[24] B.J. Leimkuhler and C.R. Sweet, The canonical ensemble via symplectic integrators using Nosé and Nosé-Poincaré chains. J. Chem. Phys. 121 (2004) 108-116.

[25] B.J. Leimkuhler and C.R. Sweet, A Hamiltonian formulation for recursive multiple thermostats in a common timescale. SIAM J. Appl. Dyn. Sys. 4 (2005) 187-216.

[26] W.K. Liu, E.G. Karpov, S. Zhang and H.S. Park, An introduction to computational nano-mechanics and materials. Comput. Meth. Appl. Mech. Eng. 193 (2004) 1529-1578.

[27] Y. Liu and M.E. Tuckerman, Generalized Gaussian Moment Thermostatting: A new continuous dynamical approach to the canonical ensemble. J. Chem. Phys. 112 (2000) 1685-1700.

[28] G.J. Martyna, M.E. Tuckerman and M.L. Klein, Nosé-Hoover chains: The canonical ensemble via continuous dynamics. J. Chem. Phys. 97 (1992) 2635-2643.

[29] S. Nosé, A unified formulation of the constant temperature molecular dynamics methods. J. Chem. Phys. 81 (1984) 511-519.

[30] J. Powles, G. Rickayzen and D.M. Heyes, Temperature: old, new and middle-aged. Mol. Phys. 103 (2005) 1361-1373.

[31] L. Rosso, P. Mináry, Z. Zhu and M.E. Tuckerman, On the use of the adiabatic molecular dynamics technique in the calculation of free energy profiles. J. Chem. Phys. 116 (2002) 4389-4402.

[32] H.H. Rugh, Dynamical approach to temperature. Phys. Rev. Lett. 78 (1997) 772-774.

[33] T. Schneider and E. Stoll, Molecular-dynamics study of a three-dimensional one-component model for distortive phase transitions. Phys. Rev. B 17 (1978) 1302-1322.

[34] J.B. Sturgeon and B.B. Laird, Symplectic algorithm for constant-pressure molecular-dynamics using a Nosé-Poincare thermostat. J. Chem. Phys. 112 (2000) 3474-3482.

[35] E.B. Tadmor, M. Ortiz and R. Phillips, Quasicontinuum analysis of defects in solids. Philos. Mag. A 73 (1996) 1529-1563.

[36] R.G. Winkler, V. Kraus and P. Reineker, Time-reversible and phase-conserving molecular dynamics at constant temperature. J. Chem. Phys. 102 (1995) 9018-9025. 